\title{
Priorização de localidades de risco quanto à Vigilância da Qualidade da Água para Consumo Humano- VIGIAGUA
}

\section{Prioritization of risky locations regarding the Monitoring of Water Quality for Human Consumption - VIGIAGUA}

\author{
DOI: $10.46919 / \operatorname{archv} 1 n 6-002$
}

Recebimento dos originais: 01/09/2020

Aceitação para publicação: 30/10/2020

\section{Glauce Araujo Ideião Lins}

Doutora em Enfermagem pela Universidade de Brasília

Instituição: Enfermeira do Trabalho e de Família da Secretaria de Estado da Saúde do Distrito federal

Endereço: AENW trecho 2 lote 4, Setor Noroeste, Brasília-DF, CEP 70684-831

E-mail: glauce.ideiao@gmail.com

\section{Kênia Cristina de Oliveira}

Mestranda em Medicina Tropical pela Universidade de Brasília/Faculdade de Medicina//Núcleo de Medicina Tropical

Instituição: Diretoria de Vigilância Ambiental em Saíde- DIVAL

Bióloga da Secretaria de Estado da Saúde do Distrito Federal

Enfermeira do Trabalho e de Família da Secretaria de Estado da Saúde do Distrito federal

Endereço: AENW trecho 2 lote 4, Setor Noroeste, Brasília-DF, CEP 70684-831

E-mail: keniacris.bio@gmail.com

\section{Petrônio da Silva Lopes}

Especialista em Gestão Ambiental- Universidade Icesp; Extensão Qualidade da Água para consumo Humano da UFRJ/MS

Instituição: Servidor do Ministério da Saúde cedido para a Diretoria de Vigilância Ambiental em SaídeDIVAL

Bióloga da Secretaria de Estado da Saúde do Distrito Federal

Endereço: AENW trecho 2 lote 4 , Setor Noroeste , Brasília-DF , CEP 70684-831

E-mail: vigiagua@gmail.com

\section{RESUMO}

A Vigilância em Saúde Ambiental Relacionada à Qualidade da Água para Consumo Humano VIGIAGUA consiste no conjunto de ações adotadas continuamente pelas autoridades de saúde pública, visando garantir que a água consumida pela população atenda aos padrões e normas estabelecidas na legislação vigente e para avaliar os riscos que a água de consumo representa para a saúde humana. Objetivou-se apresentar a metodologia de priorização de Regiões Administrativas de risco quanto à contaminação hídrica, mediante critérios relacionados às características dos sistemas e soluções de abastecimento de água, condições sanitárias e agravos de vinculação hídrica. A partir, da metodologia do planejamento do Plano de Amostragem da Vigilância da Qualidade da Água para Consumo Humano do DF, observou-se que a necessidade de elaborar estratégias locais, segundo o princípio da equidade, para cobertura de atendimento do programa VIGIAGUA-DF. Assim, obteve-se como resultado a priorização de Regiões Administrativas em três classes: de alto, médio e baixo. Logo, ratificamos a relevância de gestão do programa por meio da utilização de critérios técnicos para tomada de decisão e melhor atendimento das demandas de saúde e saneamento ambiental. 
Palavras-chave: Saúde Ambiental, Vigilância em Saúde Pública, Saúde Pública, Vigilância Sanitária Ambiental, Promoção da Saúde

\begin{abstract}
The Environmental Health Surveillance Related to the Quality of Water for Human Consumption VIGIAGUA consists of the set of actions adopted continuously by the public health authorities, aiming to ensure that the water consumed by the population meets the standards and norms established in the current legislation and to evaluate the risks that drinking water poses to human health. The objective was to present the methodology for prioritizing Administrative Regions at risk in terms of water contamination, using criteria related to the characteristics of water supply systems and solutions, sanitary conditions and water binding conditions. From the planning methodology of the Sampling Plan of the Water Quality Surveillance for Human Consumption in the Federal District, it was observed that the need to develop local strategies, according to the principle of equity, to cover the attendance of the VIGIAGUA-DF program. Thus, the prioritization of Administrative Regions in three classes was obtained as a result: high, medium and low. Therefore, we ratify the relevance of program management through the use of technical criteria for decision making and better meeting the demands of health and environmental sanitation.
\end{abstract}

Keywords: Environmental Health, Public Health Surveillance, Public Health, Environmental Health Surveillance, Health Promotion.

\title{
1 INTRODUÇÃO
}

A Vigilância em Saúde Ambiental Relacionada à Qualidade da Água para Consumo Humano VIGIAGUA consiste no conjunto de ações adotadas continuamente pelas autoridades de saúde pública, visando garantir que a água consumida pela população atenda aos padrões e normas estabelecidas na legislação vigente e para avaliar os riscos que a água de consumo representa para a saúde humana. Assim, esse trabalho justifica-se pela necessidade de planejar as ações de vigilância ambiental para água de consumo humano no território do Distrito Federal. Para tanto, formulou-se a seguinte questão norteadora: como priorizar os riscos ambientais advindos da água de consumo para a saúde humana?

\section{OBJETIVO}

Apresentar a metodologia de priorização de localidades de risco de contaminação hídrica para cobertura equânime do Programa VIGIAGUA.

\section{MÉTODO}

Durante o monitoramento do Programa VIGIAGUA-DF em 2013, observou-se que seria necessária uma análise de risco aplicada a cada Região Administrativa, devido a singularidades existentes relacionadas a fatores estruturais, sociais e de saúde. Para tanto, iniciamos em outubro de 2013, um estudo acerca de indicadores de saúde e sócio-demográficos das RA`s. 
Assim, baseando-se especialmente nos critérios elencados abaixo, que algumas regiões evidenciaram quantitativamente diferentes fatores de risco, como:

$\checkmark$ Densidade populacional;

$\checkmark$ Áreas não atendidas pelo controle;

$\checkmark$ Características inerentes aos sistemas de abastecimento (fragilidades);

$\checkmark$ Resultados das análises de amostras de água de consumo humano da vigilância e controle;

$\checkmark$ Reclamações da população quanto à qualidade da água;

$\checkmark$ Dados de saneamento do órgão de planejamento do DF - Codeplan;

$\checkmark$ Mortalidade por doenças de veiculação hídrica;

$\checkmark$ Notificação de casos suspeitos/confirmados de Hepatite A. Fonte: NATHA/DIVEP/SVS/SES-DF;

$\checkmark$ Coeficiente de incidência de Doenças Diarréicas Agudas, conforme SIVEP/MDDA Ministério da Saúde;

$\checkmark$ Dados de saneamento ambiental da Estratégia Saúde da Família (fatores de risco consumo de água não tratada e baixo \% de famílias com oferta de abastecimento público). Fonte: SIAB-DF.

Então, elencamos por grau de prioridade o conjunto de Regiões Administrativas, de acordo com o quantitativo de fatores de risco representados.

\section{PRINCIPAIS RESULTADOS ALCANÇADOS:}

Segundo Brasil (2006), a Vigilância em Saúde Ambiental Relacionada à Qualidade da Água para Consumo Humano - VIGIAGUA/ Ministério da Saúde consiste no conjunto de ações adotadas continuamente pelas autoridades de saúde pública visando garantir que a água consumida pela população atenda ao padrão e normas estabelecidas na legislação vigente e para avaliar os riscos que a água de consumo representa para a saúde humana. Suas atividades devem ser investigativas, rotineiras, preventivas e corretivas sobre os sistemas públicos e soluções alternativas de abastecimento de água, sendo realizadas e dirigidas para a identificação e correção dos fatores de risco à saúde humana associados com a água consumida.

Compreendendo que defesa do ambiente é o subproduto de uma escolha por uma vida saudável, o Distrito Federal instituiu em 2002, o Programa de Vigilância da Qualidade da Água para Consumo Humano através da capacitação de servidores, realização de cadastros, coletas e análises de amostras de água. Em 2003, foi criado o Grupo Técnico, visando à apresentação de proposta relativa ao Controle e Vigilância da Qualidade da Água Destinada ao Consumo Humano no DF; e em dezembro de 2006, foi criada a Gerência de Fatores não Biológicos - GENBIOL com o Núcleo de Vigilância da Qualidade da Água - NVQA. Em 2013, foi republicado o regimento interno da Secretaria de Estado da Saúde SES/ 
Subsecretaria de vigilância em Saúde - SVS/ Diretoria de Vigilância Ambiental em Saúde- DIVAL, designando a Gerência, como de Vigilância Ambiental de Fatores Não Biológicos - GEVANBIOL e o núcleo, como de Vigilância da Qualidade da Água para Consumo Humano NUVAG. Este Núcleo tem como atribuição a implantação e execução do Programa de Qualidade da Água para Consumo Humano no DF, conforme diretrizes nacionais estabelecidas pelo Ministério da Saúde.

Para implementação desse programa, busca-se propor ações para garantir a segurança no uso da água e a formulação de instrumentos, mecanismos e estratégias que possam contribuir na prevenção e controle dos riscos ambientais inerentes à transmissão de doenças de veiculação hídrica DVH's.

Considerando a densidade populacional em 2012, a meta alcançada foi $9 \%$ e em 2013, a pactuada foi 12\%; no entanto o DF foi considerado um município, alcançando mais de 100\%. Para 2014, o VIGIAGUA-DF pactua o avanço da meta na medida em que vai tratar para cada RA com o padrão mínimo exigido de amostras para avaliação da potabilidade (cloro residual livre, turbidez, fluoreto, coliformes totais e agrotóxicos), como exemplificada na Diretriz Nacional sobre municípios. Para tanto, foi elaborado quadro abaixo, conforme parâmetros da Diretriz Nacional do Plano de Amostragem para Vigilância da Qualidade da Água para Consumo Humano vigente em 2013 


\begin{tabular}{|c|c|c|c|c|c|c|}
\hline População residente & $\begin{array}{l}<5.000 \\
\text { hab }\end{array}$ & $\begin{array}{l}5.001 \text { a } 10.000 \\
\text { hab }\end{array}$ & 10.001 a 20.000 hab & 20.001 a 50.000 hab & 50.001 a 100.000 hab & $>100.000$ hab \\
\hline $\begin{array}{c}\text { Regiões } \\
\text { Administrativas }\end{array}$ & SIA & $\begin{array}{l}\text { Varjão } \\
\text { Fercal }\end{array}$ & $\begin{array}{l}\text { Candangolândia } \\
\text { Park Way }\end{array}$ & $\begin{array}{c}\text { Brazlândia } \\
\text { Paranoá } \\
\text { Núcleo Bandeirante } \\
\text { Cruzeiro } \\
\text { Lago Sul } \\
\text { Riacho Fundo } \\
\text { Lago Norte } \\
\text { Riacho Fundo II } \\
\text { SCIA (Estrutural) } \\
\text { Jardim Botânico }\end{array}$ & $\begin{array}{c}\text { Sobradinho } \\
\text { São Sebastião } \\
\text { Sudoeste/Octogonal } \\
\text { Sobradinho II } \\
\text { Itapoã } \\
\text { Vicente Pires }\end{array}$ & $\begin{array}{c}\text { Brasília } \\
\text { Gama } \\
\text { Taguatinga } \\
\text { Planaltina } \\
\text { Ceilândia } \\
\text { Guará } \\
\text { Samambaia } \\
\text { Santa Maria } \\
\text { Recanto das Emas } \\
\text { Águas Claras }\end{array}$ \\
\hline Parâmetros básicos & \multicolumn{6}{|c|}{$\mathrm{N}^{\circ}$ de amostras mensais de acordo com o parâmetro da Diretriz Nacional do Plano de Amostragem } \\
\hline Cloro e turbidez & 10 & 14 & $\begin{array}{c}36 \text { (mínimo de } 18 \\
\text { amostras por RA } \\
\text { mensal) }\end{array}$ & $\begin{array}{c}250 \text { (mínimo de } 25 \\
\text { amostras por RA } \\
\text { mensal) }\end{array}$ & $\begin{array}{c}216 \text { (mínimo de } 36 \\
\text { amostras por RA mensal) }\end{array}$ & $\begin{array}{c}530 \text { (mínimo de } 53 \text { amostras por } \\
\text { RA mensal) }\end{array}$ \\
\hline Fluoreto & 05 & 05 & $\begin{array}{c}10 \text { (mínimo de } 05 \\
\text { amostras por RA } \\
\text { mensal) }\end{array}$ & $\begin{array}{c}50 \text { (mínimo de } 05 \\
\text { amostras por RA } \\
\text { mensal) }\end{array}$ & $\begin{array}{c}60 \text { (mínimo de } 10 \\
\text { amostras por RA mensal) }\end{array}$ & $\begin{array}{c}680 \text { (mínimo de } 68 \text { amostras por } \\
\text { RA mensal) }\end{array}$ \\
\hline Coliformes totais & 10 & 10 & $\begin{array}{l}36 \text { (mínimo de } 18 \\
\text { amostras por RA } \\
\text { mensal) }\end{array}$ & $\begin{array}{c}250 \text { (mínimo de } 25 \\
\text { amostras por RA } \\
\text { mensal) }\end{array}$ & $\begin{array}{c}216 \text { (mínimo de } 36 \\
\text { amostras por RA mensal) }\end{array}$ & $\begin{array}{l}400 \text { (mínimo de } 40 \text { amostras por } \\
\text { RA mensal) }\end{array}$ \\
\hline Agrotóxicos & \multicolumn{6}{|c|}{$\begin{array}{c}\text { Serão coletadas em cinco bacias hidrográficas do DF, em doze comunidades produtoras, totalizando } 24 \text { amostras trimestrais e } 96 \text { amostras para análise } \\
\text { de agrotóxicos anuais. }\end{array}$} \\
\hline
\end{tabular}

Quadro 1: $\mathrm{N}^{\mathrm{o}}$ mínimo de amostras mensais, por parâmetro básico e densidade populacional, conforme preconizado na Diretriz Nacional do Plano de Amostragem do Ministério da Saúde, 2013. Fonte: GEVANBIOL/DIV 
A seguir foram avaliados conforme critérios sanitários e epidemiológicos as características das Regiões Administrativas, de acordo com a avaliação de risco à saúde por contaminação da água de consumo humano:

Alta prioridade: Para RA's que apresentam acima de três fatores de priorização: Brasília, Planaltina, Ceilândia, Taguatinga, Recanto das Emas, Estrutural, Brazlândia, Sobradinho, Sobradinho II, Fercal, Santa Maria, Jardim Botânico, São Sebastião, Paranoá, Itapoã, Gama e Park Way; realizar maior percentual, conforme $\mathrm{n}^{\mathrm{o}}$ mínimo de amostras preconizadas pela Diretriz Nacional do Plano de Amostragem do VIGIAGUA;

Média prioridade: Para RA`s que apresentam até três fatores de priorização: Samambaia, Núcleo Bandeirante, Riacho Fundo, Riacho Fundo II, Águas Claras, Guará, Varjão, Vicente Pires ; realizar percentual médio, considerando o $\mathrm{n}^{\circ}$ mínimo de amostras preconizadas pela Diretriz Nacional;

Baixa prioridade: Para RA's que apresentam até dois fatores de priorização: Sudoeste/Octogonal, Lago Sul, Lago Norte, Cruzeiro, Candangolândia, SAI, SCIA; realizar percentual mínimo, considerando o $\mathrm{n}^{\circ}$ mínimo de amostras preconizadas pela Diretriz Nacional.

\section{CONCLUSÕES E/OU RECOMENDAÇÕES PARA A SAÚDE PÚBLICA:}

Ratifica-se a relevância de atuação de forma parametrizada e com metodologia para planejar e monitorar as variáveis inerentes à potabilidade da água, utilizando os princípios do SUS de equidade e descentralização das ações para uma melhor tomada de decisão em saúde ambiental.

Assim, recomendamos trabalhar com priorização, pois otimiza recursos humanos, materiais e financeiros, além de contribuir de forma a atender as necessidades da população vulnerável. Além de facilitar o monitoramento das ações e o estabelecimento de parcerias intersetoriais, que quando realizado localmente, obtém mais efetividade na medida em que são consideradas as peculiaridades do ambiente.

Em março de 2014, foi publicada nova Diretriz Nacional do Plano de Amostragem, e foi ajustado, conforme metodologia adotada em 2013, no planejamento 2015. 


\section{REFERÊNCIAS}

Brasil. Ministério da Saúde. Subsídios para construção da Política Nacional de Saúde Ambiental. Brasília: Ministério da Saúde; 2007.

Brasil. Ministério da Saúde. Secretaria de Vigilância em Saúde. Instrução Normativa MS/SVS no 1 , de 7 de março de 2005. Regulamenta a Portaria GM/MS no 1.172/2004/GM, no que se refere às competências da União, Estados, Municípios e Distrito Federal na área de Vigilância em Saúde Ambiental. Diário Oficial da União 2005; 22 mar.

Netto GF, Carneiro FF, Aragão LGT, Bonini EM, Drumond IA, Tavares MS, Villardi JWR, Alonzo H. Saúde e Ambiente: reflexões para um novo ciclo do SUS. In: Castro A, Malo M, organizadores. SUS re-significando a promoção da saúde. $1^{\mathrm{a}}$ ed. São Paulo: Hucitec/OPAS; 2006.

Miranda AC, Barcellos C, Moreira JC, Monken M, organizadores. Território, ambiente e saúde. Rio de Janeiro: FIOCRUZ; 2008.

BRASIL. Vigilância ambiental em saúde. Brasília, DF: Funasa, 2002.

BRASIL. Ministério da Saúde. Portaria MS no 518, de 25 de março de 2004. Estabelece os procedimentos e responsabilidades relativos ao controle e vigilância da qualidade da água para consumo humano e seu padrão de potabilidade, e dá outras providências. Brasília, DF: Ministério da Saúde, 2005. (Série E, Legislação em Saúde).

Brasil. Ministério da Saúde. Manual de procedimentos de vigilância em saúde ambiental relacionada à qualidade da água para consumo humano. Brasília, DF, 2006.

Brasil. Ministério da Saúde. Diagnóstico da estrutura de controle e vigilância da qualidade da água para consumo humano: portaria MS n.518/2004. Resumo executivo. Brasília, DF, 2009. 